\title{
Role of non-HDL cholesterol in type II diabetes mellitus
}

\author{
Muralidhara Krishna C.S. ${ }^{1}$, Hemantha Kumara D.S., ${ }^{2, *}$, H.L. Vishwanath ${ }^{3}$ \\ ${ }^{\mathbf{1}}$ Assistant Professor, ${ }^{2}$ Tutor, ${ }^{3}$ Professor and HOD, Dept. of Biochemistry, ${ }^{1,3}$ Bangalore Medical College and Research Institute, \\ Bangalore, Karnataka, ${ }^{2}$ Shimoga Institute of Medical Sciences, Shimoga, Karnataka, India
}

*Corresponding Author: Hemantha Kumara D.S

Email: hemanth_kumards@yahoo.co.in

Received: $27^{\text {th }}$ February, 2018

Accepted: $25^{\text {th }}$ August, 2018

\begin{abstract}
Introduction: Type 2 diabetes mellitus is a widespread global disorder among which India is the one where $1 / 3^{\text {rd }}$ of the population suffering from diabetes and majority of them were diagnosed during the complication. Diabetic dyslipidemia is the major contributor for the cardiovascular disease. Measurement of cholesterol level without HDL (total cholesterol minus HDL cholesterol) could be a valuable indicator of this risk. The atherogenic plasma index (AIP) reveals the balance between atherosclerotic and protective lipoproteins $\{\mathrm{Log}$ (TG / HDLc) $\}$ and can also predict cardiovascular risk in patients with type II diabetes mellitus. The main aim and objective of the study is to find out the role of Non- HDL cholesterol in assessing the cardiovascular risk and also atherogenic index of plasma (AIP) ascertained as log (TG/HDL-C) can been utilized as a massive indicator of atherosclerosis and cardiovascular dangers in type II diabetes mellitus.

Materials and Methods: Case-control study included 100 (50 men and 50 women) patients with newly diagnosed type II DM in the 32-65 age group as cases and 100 (50 men and 50 women) healthy individuals of the same age group without family history Type $2 \mathrm{DM}$ of the general population as controls. All samples were studied for fasting blood glucose, total cholesterol, LDLc, HDLc and Triglycerides, while non-HDL cholesterol and AIP levels were calculated.

Results: The present study showed that there was a significant rise in Non-HDLc $(p<0.0001)$ and AIP (p <0.0001) in cases than controls. Non HDL cholesterol showed a moderate positive statistical correlation with total cholesterol $(\mathrm{r}=0.7362)$ and LDLc $(\mathrm{r}$ $=0.6374$ ).

Conclusion: Non-HDL cholesterol and AIP can be used as indicators of diabetic dyslipidemia and also be used to predict the risk of cardiac and vascular disease in type $2 \mathrm{DM}$.
\end{abstract}

Keywords: Non-HDLc (Non HDLc cholesterol), AIP (Atherogenic index of plasma), LDLc (LDL cholesterol), HDLc (HDL cholesterol).

\section{Introduction}

Diabetes mellitus (DM) is a one of the major global disorder; characterized by long standing hyperglycemia due to absolute or relative deficiency of insulin secretion or its action. ${ }^{1}$ Insulin; is a protein polypeptide, hormone that regulates the body's use of glucose; and is released by the $B$ cells of islets of Langerhans; present in the pancreas. ${ }^{2}$ Mainly there are two types of classification; Type I (Insulin dependent) and Type II (Non-Insulin dependent).

Type II DM is a solemn global; health problem where $85 \%$ to $95 \%$ of diabetics are in high-income countries and around $80 \%$ are in low and middleincome countries. ${ }^{3}$ There is generous evidence that it is epidemic in many economically developing and newly mechanized countries like India. ${ }^{4}$ Type II DM is a heterogeneous; disease which is characterized; by capricious degrees of insulin resistance and increased glucose production. Insulin resistance occurs when the cells become less sensitive; to the effects of insulin. Type II DM; is a chronic and progressive disease. It impedes the quality of life of the patients and also leads to death due to micro and macro-vascular complications. Age adjusted mortality rates among diabetics is 1.5 to 2.5 times greater than general population. ${ }^{2,5}$
Most of the Type II Diabetics remain unaware; of their illness for a long time because symptoms may take months - years to appear or be recognised, during which time the body is being rotted by excess blood glucose. They are often diagnosed only when complications of diabetes have already advanced. When compared to type I diabetics, the majority of those with Type II DM usually do not require daily doses of insulin to survive. Many people are able to manage their condition through a healthy nutritious balanced diet and moderate physical activity; or oral medication. However, if they are unable to normalize their blood glucose levels by above methods, patients are put on insulin therapy.

All the developing and developed nations are from the impacts of the diabetes epidemic..$^{5}$ It has turned out to be one of the world's most vital' general medical issues and records for $85-90 \%$ of diabetes around the world. Most of the 382 million individuals with diabetes are matured in the vicinity of 40 and 59, and $80 \%$ of them live in low-and center pay nations. A wide range of DM are on the expansion, Type II DM specifically, the quantity of individuals with Type II DM will increment by $55 \%$ by $2035 .{ }^{5}$ It ordinarily happens in adults (>40 years), however now it is additionally progressively found in kids and adolescents. 5 The plague is related with financial advancement, maturing populaces, expanding 
urbanization, dietary changes, lessened physical movement, and changes in other way of life designs.

Among the different variables for creating expanded cardiovascular hazard in diabetes; the dyslipidemia; is the major contributor. The normal sort of dyslipidemia in diabetics is described by raised Triglyceride (TG), low levels of high density lipoprotein cholesterol (HDL-C). ${ }^{6}$

National Cholesterol Education Program Treatment for adult's section III (NCEP ATP III) expresses the level of LDL-C is a significant reason for repair purposes at TG level over $200 \mathrm{mg} / \mathrm{dL}$, Non-HDL cholesterol (non HDL-C); is identified as the corrective target at that level of TG. ${ }^{7}$ Non-HDL cholesterol (non HDL-C) is such a marker, the point that is obtained by subtracting HDL cholesterol from total cholesterol. ${ }^{8}$ Estimating non-HDL-C could be more illustrative than all lipoproteins containing atherogenicapolipoprotein $\mathrm{B}$ (apoB) LDL - C, lipoprotein, VLDL-C, lipoprotein cholesterol mediator (IDL-C) and lipoprotein (a). ${ }^{9,10}$ In patients with type 2 diabetes, the level of LDL-C; it can not be lifted significantly, so the cardiovascular risk is not precisely identified. This increases the way in which the importance of computing non-HDL-C; It is particularly important and may be supplemented by an additional device for assessing cardiovascular dangers. ${ }^{11} \mathrm{~A}$ considerable measure of work has been done on the connection amongst TG and HDL-C, and it has been demonstrated that the proportion of TG to HDL-C was a solid indicator of myocardial infarction. ${ }^{11}$ All around, atherogenic index of plasma (AIP) ascertained as $\log$ (TG/HDL-C) has been utilized by a few experts as a massive indicator of atherosclerosis and cardiovascular dangers. ${ }^{12,13}$

\section{Materials and Methods}

The study comprises of 100 (50 males and 50 females) newly diagnosed type 2 DM patients of age group 32-65 years in Victoria Hospital, attached to Bangalore Medical College and Research Institute, Bangaluru and 100(50 males and 50 females) healthy

individuals of same age group with no family history of type 2 DM from general population. This is a casecontrol study conducted in Victoria Hospital, attached to Bangalore Medical College and Research Institute, Bangaluru. Type 2 diabetes mellitus cases which are recently diagnosed and age group between 30 and 65 years, Type 2 Diabetics diagnosed conferring to the ADA criteria are included in this study. Age and sex matched healthy subjects are considered as controls. Patients already on treatment for diabetes mellitus, liver dysfunction, thyroid disorders and Pregnant and lactating mothers are excluded from the study.

\section{Methodology}

After obtaining written informed consent from the cases and controls, about $5 \mathrm{ml}$ of fasting venous blood was obtained by venepuncture under aseptic conditions and divided into 2 parts, first volume of sample was taken in a sterile EDTA tube and was used for measuring $\mathrm{HbA} 1 \mathrm{C}$ and second volume of sample in a plain tube, centrifuged and separated serum was used for measuring fasting blood glucose and Lipid profile. Parameters were measured in BECKMAN COULTER AU480 including HbA1c. Non-HDL cholesterol was also calculated.

\section{Statistical Analysis}

The results were tabulated. Results on continuous measurements are presented on Mean \pm SD. The Statistical analysis of data was done by using software namely SAS 9.2, SPSS 20.0, Stata 10.1, MedCalc 9.0.1, Systat 12.0 and R env ver.2.1.1. The results of cases and controls were compared by student' $t$ ' test. A ' $p$ ' value of $<0.05$ was considered significant. A ' $p$ ' value of $<0.0001$ was considered as highly significant. Pearson correlation between study variables is performed to find the degree of relationship.

\section{Results \\ Results}

Table 1: Age distribution in the cases and controls studied

\begin{tabular}{|c|c|c|c|}
\hline Age group (in years) & Controls & Cases & Total \\
\hline $30-40$ & 21 & 27 & 48 \\
\hline $41-50$ & 39 & 33 & 72 \\
\hline $51-60$ & 33 & 30 & 63 \\
\hline $61-65$ & 07 & 10 & 17 \\
\hline
\end{tabular}

Table 2: Comparison FBG and Hba1c levels in T2DM cases and controls

\begin{tabular}{|l|c|c|c|}
\hline \multicolumn{1}{|c|}{ Parameters } & $\begin{array}{c}\text { Cases }(\mathbf{n}=\mathbf{1 0 0}) \\
\text { Mean } \pm \text { SD }\end{array}$ & $\begin{array}{c}\text { Controls(n=100) } \\
\text { Mean } \pm \text { SD }\end{array}$ & p \\
\hline Fasting Blood Glucose $(\mathrm{mg} / \mathrm{dl})$ & $188.23 \pm 26.78$ & $95.65 \pm 9.65$ & $<0.0001$ \\
\hline HbA1 $\mathrm{C}$ & $7.27 \pm 0.81$ & $4.92 \pm 0.25$ & $<0.0001$ \\
\hline
\end{tabular}


Table 3: Lipid profile in T2DM cases and controls

\begin{tabular}{|l|c|c|c|}
\hline \multicolumn{1}{|c|}{ Lipid profile } & $\begin{array}{c}\text { Cases(n=100) } \\
\text { Mean } \pm \text { SD }\end{array}$ & $\begin{array}{c}\text { Controls(n=100) } \\
\text { Mean } \pm \text { SD }\end{array}$ & P \\
\hline $\begin{array}{l}\text { Total Cholesterol } \\
(<200 \mathrm{mg} / \mathrm{dl})\end{array}$ & $204.87 \pm 35.58$ & $143.93 \pm 40$ & $<0.0001$ \\
\hline $\mathrm{LDL}(<100 \mathrm{mg} / \mathrm{dl})$ & $121.74 \pm 35.86$ & $86.49 \pm 33.05$ & $<0.0001$ \\
\hline $\mathrm{HDL}(<50 \mathrm{mg} / \mathrm{dl})$ & $39.21 \pm 10.67$ & $37.92 \pm 14.32$ & 0.4709 \\
\hline VLDL $(<40 \mathrm{mg} / \mathrm{dl})$ & $40.68 \pm 21.09$ & $25.76 \pm 11.29$ & $<0.0001$ \\
\hline Triglycerides $(<150 \mathrm{mg} / \mathrm{dl})$ & $196.57 \pm 91.10$ & $122.76 \pm 45.96$ & $<0.0001$ \\
\hline
\end{tabular}

Table 4: Non-HDL and AIP levels in T2DM cases and controls

\begin{tabular}{|l|c|c|c|}
\hline \multicolumn{1}{|c|}{ Lipid Indices } & $\begin{array}{c}\text { Cases(n=100) } \\
\text { Mean } \pm \text { SD }\end{array}$ & Controls(n=100) Mean \pm SD & P \\
\hline Non-HDL & $166.57 \pm 33.84$ & $106.53 \pm 24.46$ & $<0.0001$ \\
\hline AIP & $0.66 \pm 0.25$ & $0.50 \pm 0.24$ & $<0.0001$ \\
\hline
\end{tabular}

\section{Discussion}

In the present study, Table 1 , most cases and controls belong to the age group of $41-50$ years. In the table 2, FBG and HbalC levels were higher in cases and within normal limits in controls and were statistically significant. In Table 3, the levels of the serum lipid profile showed a significant increase in total cholesterol, LDL, VLDL and triglyceride levels in cases compared to controls.

The study by Sniderman et al. explains that slight disruption in metabolism of carbohydrate due to diabetes progress to a trouble in metabolism of lipids. It certainly affects lipid levels in the body and it has been proven by many research studies. Therefore, due to abnormal metabolism, there is a much more nuclealization of cholesterol and triglycerides. This leads to reduced HDL cholesterol in the blood. In this study, our aim was to investigate the role of non-HDL cholesterol in the diabetic population.

Our study revealed that Non HDL cholesterol and AIP levels were significantly rised in diabetics than non-diabetics. And also our study showed that nonHDL cholesterol level has moderate positive correlation with total cholesterol (r value $=0.7362$ ) and LDL cholesterol $(\mathrm{r}$ value $=0.6374)$.

In Table 3 and 4, our found results were similar to the findings of Aryaali et al. (2010-11) ${ }^{14}$ and S Zabeens et al. (2012-14). ${ }^{15}$ Both studies mentioned that HDL levels were not associated with the higher risk of atherosclerosis in type $2 \mathrm{DM}$ and could be a basic and additional marker for detecting the cardiovascular disease. Due to this, the non-HDL cholesterol incorporates an evaluation of all lipoproteins considered atherogenic.

Non HDL cholesterol is a calculated marker obtained by subtracting the level of HDL cholesterol from the total cholesterol. This calculated parameter measures LDL cholesterol and other apoproteins B particles for example, VLDL, IDL and chylomicron residues. The apo-B level can be obtained directly but estimate is not widely accessible in most of the diagnostic laboratories. In both cases, the estimation of non-HDL-C is more reliable, accessible, adoptable even in small laboratories and is considered as a replacement or additional marker for apo-B in daily routine clinics.

The National Cholesterol Education Program (NCEP) for Adults III Panel emphasizes that non-HDL cholesterol might be a lethal indicator of atherosclerosis in type II DM. ${ }^{16}$ Type 2 DM have regularly increased the level of triglycerides, however, the normal estimate of LDL and HDL is not useful for assessing the risk of atherosclerosis and controlling the treatment.

\section{Conclusion}

From the results of the present study, it may be concluded that type 2 diabetes mellitus is associated with a significant rise in non-HDL and AIP levels compared to non diabetic controls. The non-HDL cholesterol is more distinguishing than all atherogenic lipoproteins and AIP is a significant predictor of atherosclerotic damage. The non-HDL cholesterol and AIP can be considered as marker of diabetic dyslipidemia. Even the both calculated parameters can be considered for assessing the cardiovascular risk in type 2 diabetic patients. The Non-HDL cholesterol is more reliable, accessible, adoptable even in small laboratories.

\section{References}

1. Peter H. Bennett, Williams C. Knowles. Definition, diagnosis and classification of diabetes mellitus and glucose homeostasis. Smith, Josln's Diabetes Mellitus.14th edition. Boston: Ovid Technologies, Inc.;2005;331-333.

2. K Park. Diabetes mellitus, Park`s text book of preventive and social medicine, 24t ed. 2013;341-345.

3. Alvin C. Powers, Longo, Fauci, Kasper, Hauser, Jameson et al. Diabtes Mellitus Harrison's Principles of Internal Medicine. 18thed. New York: McGraw-Hill Medical publishing division; 2012;2968-74.

4. WHO -World Health Organisation report - The 10 leading causes of death in the world, 2000 and 2012 , revised in May 2014.

5. The global burden, International Diabetes Federation 6th ed. $2013 ; 12,22,16,14,33-34$. 
6. Sniderman AD, Scantlebry T, Cianflon K. Hypertriglyceridemia, apoB: the unappreciated atherogenicdyslipoproteinemia in type II diabetes mellitus. An Intern Med. 2001;135:447-59.

7. Executive summary; of the $3^{\text {rd }}$ report of the National Cholesterol Education Program (NCEP) expert panel on detection, evaluation, and treatment of high cholesterol in adults (Adult Treatment Panel III). J Amr Med Asso. 2001;285:2486-97.

8. Jiang R, Schulze M, Li T. 'Non-HDL cholesterol and apoprotein B predict cardiovascular disease events among men with type II diabetes'. DM care. 2004;27:1992-1997.

9. Grunday SM. Low-density lipoprotein, nonhigh-density lipoprotein, and apolipoprotein B as goals of lipidlowering therapy. Circulation 2002;106:2526-9.

10. Peters AL. Clinical relevance of Non- HDL cholesterol in patients with diabetes Mellitus. Clin DM. 2008;26:3-7.

11. Lu W, Resniick HE, Jablonskii KA. Non-HDL cholesterol as a predictor of cardiovascular disease in type II diabetics: the Strong Heart Study. Diabetes Care world. 2003;26:16-23.

12. The expert Panel: Third report of National Cholesterol Education Program (NCEP) Expert Panel on Detection, Evaluation and Treatment of high cholesterol in Adults (Adult Treatment Panel III): final report. Circulation. 2002;106:3143-3421.
13. Lamarche B, Despres JP, Moorjani S, Cantin B, Dagenais GR, Lupien PJ. Prevalence of dyslipidemic phenotypes in ischemic heart disease (prospective results from the Quebec Cardiovascular study. Am J Cardiol. 1995;75:1189-1195.

14. Aryal M, Poudel A, Satyal B and Gayawali P, Pokheral BR, Rautt BK, Adhkari RK, et. al; Evaluation of nonHDL cholesterol and total cholesterol: HDL cholesterol ratio as Cumulative Marker of Cardiovascular Risk in Diabetes Mellitus, Kathmandu University Medical Journal. 2010;332-338.

15. Zabeen S, Reahman MR, Mustafaa TG, Eusufzai NH and Shermin S, Non-HDL Cholesterol and Type II Diabetes Mellitus, AKMMC J. 2012;3(2):15-18.

16. Abbassi MA, Hafeezullah, Shah NA. 'Non-high density lipoprotein cholesterol and type II Diabetes mellitus.' Pak J Physiol. 2007;2-3.

How to cite this article: Muralidhara K.C.S, Hemantha K.D.S, Vishwanath H.L. Role of nonHDL cholesterol in type II diabetes mellitus. Int J Clin Biochem Res. 2018;5(4):642-645. 